\title{
Autorenverzeichnis «Medizinische Onkologie: Neue Medikamente - Hoffnungsträger mit Risiken»
}

\section{David Cella, PhD}

Center for Healthcare Studies

Institute for Public Health and Medicine,

Preventive Medicine-Health

and Biomedical Informatics,

Psychiatry and Behavioral Sciences

and Weinberg College of Arts and Sciences

Department of Medical Social Sciences

633 N. St. Clair, Suite 1900

Chicago IL 60611, USA

Prof. Dr. med. Mathias Freund

Klinik für Innere Medizin III

Hämatologie, Onkologie, Palliativmedizin

Zentrum für Innere Medizin

Universitätsmedizin Rostock

Ernst-Heydemann-Straße 6

18055 Rostock, Deutschland

Prof. Dr. med. Andreas Hochhaus

Abt. Hämatologie/Onkologie

Klinik für Innere Medizin II

Universitätsklinikum Jena

Erlanger Allee 101

07740 Jena, Deutschland

PD Dr. med. Diana Lüftner

Medizinische Klinik mit Schwerpunkt Hämatologie,

Onkologie und Tumorimmunologie

Campus Benjamin Franklin

Charité - Universitätsmedizin Berlin

Hindenburgdamm 30

12200 Berlin, Deutschland

Prof. Dr. med. Andreas Mackensen

Medizinische Klinik 5 -

Hämatologie und Internistische Onkologie

Universitätsklinikum Erlangen

Ulmenweg 18 (Internistisches Zentrum)

91054 Erlangen, Deutschland
Dr. med. Friedrich Overkamp

Praxis und Tagesklinik für Internistische Onkologie

Springstraße 24

45657 Recklinghausen, Deutschland

Dr. med. Klaus-Peter Thiele

Kompetenz Centrum Onkologie

MDK Nordrhein

Bismarckstraße 43

40210 Düsseldorf, Deutschland

Dr. med. vet. Volker Vervölgyi

Institut für Qualität und Wirtschaftlichkeit im

Gesundheitswesen (IQWiG)

Im Mediapark 8

50670 Köln, Deutschland

Prof. Dr. med. Martin Wilhelm

Medizinische Klinik 5

Klinikum Nürnberg

Prof.-Ernst-Nathan-Straße 1

90419 Nürnberg, Deutschland

Prof. Dr. med. Bernhard Wörmann

Medizinischer Leiter

DGHO Hautpstadtbüro

Alexanderplatz 1

10178 Berlin, Deutschland 